\section{THU0531 "BOTHERING TO LISTEN": ENABLING PATIENT AND FAMILY INVOLVEMENT IN CHILDHOOD UVEITIS HEALTHCARE}

Sandrine Compeyrot-Lacassagne ${ }^{1,1}$, Christine Twoney ${ }^{1}$, Harry Petrushkin ${ }^{2,3}$ Dhanes Thomas ${ }^{3}$, Emily Robinson ${ }^{4}$, Lucia Kossarova ${ }^{1}$, Ameenat Lola Solebo ${ }^{2}$. ${ }^{1}$ Great Ormond Street Hospital for Children, Rheumatology, London, United Kingdom; ${ }^{2}$ Great Ormond Street Hospital for Children, Ophthalmology, London, United Kingdom; ${ }^{3}$ Moorfields eye hospital, Ophthalmology, London, United Kingdom; ${ }^{4}$ Great Ormond Street for Children, Biomedical Research Centre, London, United Kingdom

Background: Childhood uveitis is a group of heterogenous, potentially blinding inflammatory disorders. Management is complex. There is growing recognition of the importance of actively involving affected children and their families in their own care. Co-designed interventions, developed through active involvement of staff and patients, can provide effective solutions to problems identified by those affected.

Objectives: To use findings from a patient and family discussion group to inform the co-designed development of health care processes and interventions.

Methods: Five children/young people with uveitis (age ranges 8 to 17), seven parent/carers of children with uveitis and four health care professionals attended a 90 minute discussion group. Main discussion topic was the identification of areas in need of interventions or support structures. Sub-topics were determined a priori using previous PPI and existent research (REFS). They comprised: Direct health care; Impact on families; School, education and peers. Responses were collated. Consent was taken for use of direct quotes from participants.

Results: We outline the areas identified by children and families:

Direct health care: Four interconnected areas were identified: (1) Transitioning to adult services, (2) peer support (health care services being a valuable site for identifying peers), (3) communication between care structures, and (4) education for families. With regards to family education, there was identification of the need for specific services or interventions around the communication of (4a) diagnosis, (4b) treatment, (4c) likely long term outcomes/prognosis, (4d) and the child's progress. There was also discussion on (4e) the formats used to communicate with families. Impact on families: Participants discussed support around (1) family relationships, (2) the impact of systemic medication. They also discussed (3) a need for recognition of the changing nature of their lived experience as affected families over the disease course and the need for on-going psychologic support especially at presentation to help with acceptance. School, education and peers

Participants discussed the need for support around: (1) the impact of treatment on school life, (2) communication with school professionals and peers, (3) impact of visual impairments, (4) managing the visibility/invisibility paradox (ie being made to feel different but also having a disorder which was not externally apparent), and (5) managing adolescence.

Conclusion: Through the above approach, we have identified a range of issues affecting our patients and their families. Our findings are similar to those of other groups $(1,2)$ These lived experiences will be used to inform the co-design of supportive services (patient leaflets, videos, website, psychology intervention) and research on the effectiveness of these interventions in improving the management of affected children and their families.

\section{REFERENCES:}

[1] Parker DM, Angeles-Han ST, Stanton AL, Holland GN. Chronic Anterior Uveitis in Children: Psychosocial Challenges for Patients and Their Families. Am J Ophthalmol. 2018 Jul; 191:xvi-xxiv

[2] Silva LMP, Arantes TE, Casaroli-Marano R, Vaz T, Belfort R Jr, Muccioli C. Quality of Life and Psychological Aspects in Patients with Visual Impairment Secondary to Uveitis: A Clinical Study in a Tertiary Care Hospital in Brazil. Ocul Immunol Inflamm. 2017 Oct 11:1-9.

Disclosure of Interests: Sandrine Compeyrot-Lacassagne Grant/research support from: Abbvie, Christine Twoney: None declared, Harry Petrushkin: None declared, Dhanes Thomas: None declared, Emily Robinson: None declared, Lucia Kossarova: None declared, Ameenat Lola Solebo: None declared

DOI: 10.1136/annrheumdis-2019-eular.7262

\section{THU0532 RITUXIMAB FOR RAPIDLY PROGRESSIVE JUVENILE SYSTEMIC SCLEROSIS}

Roberto Dal Pozzolo ${ }^{1}$, Alessandra Meneghel ${ }^{1}$, Biagio Castaldi ${ }^{1}$, Giovanni Civieri ${ }^{1}$, Giorgia Martini', Renzo Marcolongo ${ }^{2}$, Alida Linda Patrizia Caforio ${ }^{3}$,

Francesco Zulian'. ' 'University Hospital of Padova, Department of Woman's and

Child's Health, Padova, Italy; ${ }^{2}$ University Hospital of Padova, Division of

Hematology and Clinical Immunology, Padova, Italy; ${ }^{3}$ University Hospital of

Padova, Department of Cardiac, Thoracic and Vascular Sciences, and Public health, Padova, Italy

Background: Juvenile Systemic Sclerosis (JSSc) is a rare multi-systemic disease characterized by fibrous changes of the skin and internal organs [1]. Patients with "rapidly progressive SSc" usually present rapid development of skin induration and important organ damage [3], leading to poor prognosis [2, 3]. Recently Rituximab (RTX), a monoclonal antibody against the CD20 antigen on B cells, has demonstrated to be a promising therapy for adult patients with SSc $[4,5]$.

Objectives: We describe four pediatric patients with rapidly progressive JSSc treated with RTX.

Methods: Data on clinical, laboratory and instrumental parameters were collected from four patients with rapidly progressive JSSc treated with RTX for at least one year. Data were recorded at baseline and every 6 months after initiation of therapy. All patients underwent i.v. RTX therapy with four cycles $\left(375 \mathrm{mg} / \mathrm{m}^{2}\right.$ every 2 weeks), at 3 months intervals. Oral prednisone (PDN, $0.5 \mathrm{mg} / \mathrm{Kg} / \mathrm{day}$ ) and oral mycophenolate mofetil (MMF, $500 \mathrm{mg} / \mathrm{m}^{2} /$ day) were administered between RTX pulses. Skin changes were assessed by MRSS, changes on muscles involvement by CMAS. Variations on BMI, pulmonary function tests (FVC, FEV1, DLCO) and cardiac involvement (LVEF, LVEDV, GLS) were expressed as\% change from baseline. J4S was used to assess the overall disease severity [6]

Results: Four JSSc patients (3M, 1F), aged 8-17 years, entered the study. Three patients presented with prevalent cardiological involvement. One patient (Case 3) presented with severe pulmonary involvement. After one year RTX treatment, all patients showed significant decreased of number/duration of Raynaud Phenomenon attacks and 3 patients of cutaneous involvement. Two patients needed an implantable cardioverter defibrillator (ICD) because of episodes of severe ventricular tachycardia (VT). After 12 months of therapy one patient presented improvement LVEF $(+19 \%)$ and J4S, the other showed a global cardiac improvement (LVEF $+37 \%$, LVEDV -18\%) and J4S. Both underwent a second yearlong treatment with RTX with no worsening of internal organs' involvement. Case 3 showed a significant improvement of the respiratory function (FVC $+46 \%$, FEV1 $+33 \%$, DLCO $+30 \%$ ) with decrease of J4S. Case 4 improved her arrhythmia and muscle strength (CMAS $+17 \%)$. No major RTX-related side effects were reported.

Conclusion: Rapidly progressive JSSc still carries a high mortality rate To the best of our knowledge this is the first case series of patients with JSSc successfully treated with RTX. Our experience, although in a small cohort, confirms the beneficial effect of this therapy on the lifethreatening internal organ involvement, particularly on cardiac function.

\section{REFERENCES :}

[1] Martini G, et al. Arthritis Rheum 2006; 54(12): 3971.

[2] Martini G, et al. Rheumatology (Oxford) 2009;48(2):119.

[3] Joven BE, et al. Semin Arthritis Rheum. 2010; 39:285.

[4] Daoussis D, et al. Semin Arthritis Rheum 2017; 46 (5): 625-31.

[5] Jordan S, et al. Ann Rheum Dis, 2015; 74: 1188-94.

[6] La Torre F, et al. Arthritis Rheum. 2012;64(12):4143-50

Disclosure of Interests: None declared

DOI: 10.1136/annrheumdis-2019-eular.7377

\section{THU0533 IMPAIRED PLATELET FUNCTIONS IN PATIENTS TREATED WITH COLCHICINE}

Özlem Şatırer ${ }^{1}$, Selcan Demir ${ }^{2}$, Erdal Sag ${ }^{2}$, Armağan Keskin ${ }^{1}$, Yelda Bilginer ${ }^{2}$, Şule Ünal ${ }^{3}$, Seza Özen ${ }^{2} .{ }^{1}$ Hacettepe University Faculty of Medicine, Pediatrics, Ankara, Turkey; ${ }^{2}$ Hacettepe University Faculty of Medicine, Pediatric Rheumatology, Ankara, Turkey, ${ }^{3}$ Hacettepe University Faculty of Medicine, Hacettepe University Center for Fanconi Anemia and Other Inherited Bone Marrow Failure Syndromes, Ankara, Turkey

Background: Colchicine has been used in the treatment of Familial Mediterranean Fever (FMF) since 1972. Apart from the inhibiting mitosis in all cells, colchicine has an anti-inflammatory effect by inhibiting activation and migration of neutrophils. Colchicine is a safe drug at recommended 